\title{
EVOLUCIÓN DE LA DESNUTRICIÓN CRÓNICA EN MENORES DE CINCO AÑOS EN EL PERÚ
}

\begin{abstract}
José Sánchez-Abanto $1, a$
RESUMEN

La desnutrición crónica infantil constituye uno de los principales problemas de Salud Pública en el Perú, según los valores de referencia de la OMS, la prevalencia nacional es del 19,5\% en niños menores de cinco años. La desnutrición crónica infantil afecta negativamente al individuo a lo largo de su vida, limita el desarrollo de la sociedad y dificulta la erradicación de la pobreza. Para lograr la meta de reducir a 10\% la desnutrición crónica infantil para el año 2016, el Gobierno peruano deberá continuar fortaleciendo principalmente el uso eficiente de recursos económicos, la evaluación de intervenciones, la realización de investigaciones que permitan definir relaciones de causalidad y brindar información para el diseño de políticas públicas, el fortalecimiento de las capacidades de recursos humanos en salud y la articulación de los diferentes niveles de Gobierno. En el artículo se revisan las principales intervenciones realizadas en el país.
\end{abstract}

Palabras clave: Desnutrición; Agenda de prioridades en salud; Desnutrición infantil; Programas nacionales de salud; Perú (fuente: DeCS BIREME).

\section{EVOLUTION OF CHRONIC MALNUTRITION IN CHILDREN UNDER FIVE IN PERU}

\begin{abstract}
Child chronic malnutrition is one of the main problems of Public Health in Peru, according to WHO's reference values, national prevalence is $19.5 \%$ in children under five. Child chronic malnutrition has a negative impact on people throughout their lives, limits the development of society and hinders poverty eradication. To attain the goal of reducing chronic malnutrition in children to $10 \%$ by 2016 , the Peruvian government will continue to strengthen, mainly, the efficient use of economic resources, the assessment of interventions, the implementation of investigations that help define causal relations and provide information for the design of public policies, health capacity building and the articulation of different government levels. This article goes over the main interventions implemented in the country.
\end{abstract}

Key words: Malnutrition; Health priority agenda; Child nutrition disorders; National health programs; Peru (source: MeSH NLM).

\section{INTRODUCCIÓN}

La prevalencia de desnutrición crónica infantil es un indicador utilizado por todos los países para vigilar los logros de las intervenciones en salud y nutrición. La desnutrición crónica infantil constituye uno de los principales problemas de Salud Pública en el Perú, que afecta al $19,5 \%$ de niños menores de cinco años. Esta condición se acentúa en la población de más temprana edad y con mayor grado de exclusión, como es el caso de la población rural, de menor nivel educativo y de menores ingresos económicos. En 16 departamentos del país existen cifras superiores a la media nacional, mientras que en siete de ellos, las prevalencias superan el $30 \%{ }^{(1)}$, punto de corte internacional establecido por la OMS para definir a la desnutrición crónica infantil como un problema de Salud Pública de alta prevalencia (2). Esto demuestra el alto grado de desigualdad e inequidad existente en el Perú.

La desnutrición crónica infantil es un fenómeno de origen multifactorial, resultado de una amplia gama de condiciones sociales y económicas. Dentro de las causas inmediatas relacionadas con su desarrollo

\footnotetext{
Dirección Ejecutiva de Vigilancia Alimentaria y Nutricional, Centro Nacional de Alimentación y Nutrición, Instituto Nacional de Salud. Lima, Perú.

Nutricionista magíster en Nutrición

Recibido: 24-07-12 Aprobado: 29-08-12

Citar como: Sánchez-Abanto J. Evolución de la desnutrición crónica en menores de cinco años en el Perú. Rev Peru Med Exp Salud Publica. 2012;29(3):402-5.
} 
figura la ingesta inadecuada de nutrientes y las enfermedades de tipo infeccioso (especialmente las enfermedades respiratorias y gastrointestinales) (3). Asimismo, existen condiciones sociales íntimamente relacionadas con su desarrollo, como son el bajo nivel educativo de la madre; la alimentación deficiente en calidad y cantidad; las condiciones inadecuadas de salud y saneamiento, y el bajo estatus social de la madre en la toma de decisiones dentro del hogar (4). Además, la desnutrición crónica infantil genera daños permanentes e irrecuperables después del segundo año de vida en nuestra población ${ }^{(5,6)}$.

En el siguiente artículo se presenta una descripción del estado de la desnutrición crónica infantil en Perú, las principales intervenciones realizadas en el Estado y las nuevas estrategias dirigidas para cumplir con los objetivos del actual Gobierno.

\section{LAEVOLUCIÓNDELADESNUTRICIÓN CRÓNICA INFANTIL EN EL PERÚ}

En el año 1996, la prevalencia de desnutrición crónica infantil en el Perú era de $25,8 \%$ de niños menores de cinco años (Patrón NCHS) ${ }^{(7)}$. Esta cifra se mantuvo prácticamente inalterable por espacio de casi una década. Durante la década del 2000 la prevalencia de desnutrición crónica infantil mostró una reversión significativa, especialmente entre los años 2007 y 2010 , mostrando a partir de la fecha y hasta la actualidad una disminución progresiva, reducción mayormente observada en el área rural (de $45,7 \%$ en el año 2007 a $37 \%$ en el año 2011), y en los departamentos de la sierra del país (de 42,4\% en el año 2007 a $30,7 \%$ en el año 2011), como se ve en la Figura 1, y que obedece a los resultados obtenidos a políticas sociales dirigidas al sector rural ${ }^{\left({ }^{8}\right)}$. Estos resultados son consistentes con las tendencias, obtenidos por el Sistema de Información del estado nutricional (SIEN) realizado por el Instituto Nacional de Salud (INS), sobre la base de la población infantil menores de cinco años, que acude a los establecimientos de salud públicos del Perú.

Considerando la población de referencia americana (NCHS) se observa una caída de $9 \%$ en la prevalencia de la desnutrición crónica entre el 2005 al 2011. Si se usa la nueva población de referencia de la OMS, la caída es del $10 \%$ en el mismo periodo. Respecto al SIEN, la tendencia es menor a $3 \%$, debido a que es un subgrupo poblacional de los niños menores de cinco años que acceden a los servicios de salud, siendo los niños del entorno urbano, confirmándonos que la disminución de la desnutrición crónica se ha realizado en forma más significativa en el área rural.

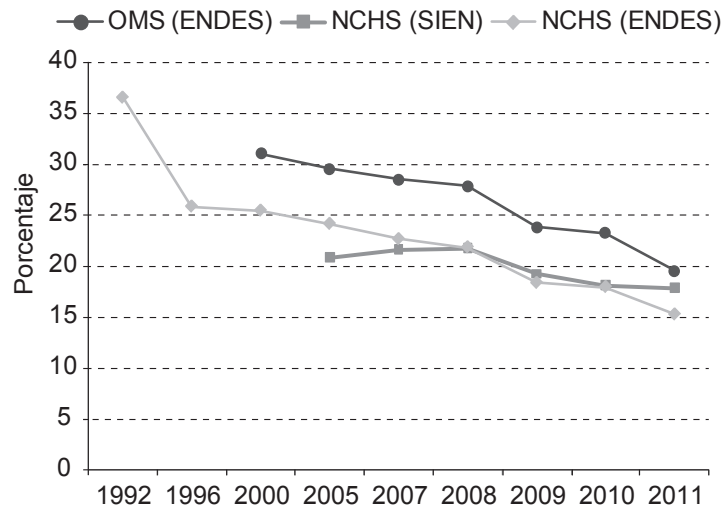

Figura 1. Evolución de la desnutrición crónica infantil en niños menores de cinco años.

Fuentes: Encuesta Demográfica y de Salud Familiar, Instituto Nacional de Estadística e Informática; Sistema de Información del estado nutricional, Instituto Nacional de Salud.

\section{PRINCIPAL INTERVENCIÓN EN LA REDUCCIÓN DE LA DESNUTRICIÓN CRÓNICA INFANTIL EN EL PERÚ}

\section{ESTRATEGIA NACIONAL CRECER}

Estrategia creada en el año $2007{ }^{(9)}$ con la finalidad de lograr la articulación de las entidades públicas (gobierno local, regional y local), las entidades privadas, de cooperación internacional y de la sociedad civil, para mejorar las condiciones de vida de la población en pobreza y pobreza extrema, así como disminuir la desnutrición crónica infantil. Actualmente, interviene en distritos pobres de la sierra rural y distritos urbanos con mayores niveles de desnutrición crónica, alcanzando una cobertura de 1119 distritos. Las intervenciones de CRECER se enfocan en mejorar las condiciones de agua, saneamiento y prácticas de alimentación y nutrición, y tratamiento de enfermedades diarreicas y respiratorias.

Esta estrategia ha permitido involucrar componentes productivos en el desarrollo de los programas sociales, como mayor capacitación, mejores prácticas de alimentación, mejores hábitos de higiene, mayor acceso a agua segura, y uso de transferencias condicionadas de efectivo para apoyar la reducción de la desnutrición. Asimismo, se promovió la complementariedad y descentralización de intervenciones, transfiriendo paulatinamente responsabilidades administrativas, financieras y políticas a los gobiernos regionales y municipales ${ }^{(10)}$.

Cabe mencionar que aún necesitan ser fortalecidos aspectos de asistencia técnica y acompañamiento para 
Tabla 1. Evolución de los resultados intermedios y productos del PAN.

\begin{tabular}{lrrrrr}
\hline \multicolumn{1}{c}{ INDICADORES } & $\mathbf{2 0 0 7}$ & $\mathbf{2 0 0 9}$ & $\mathbf{2 0 1 0}$ & $\mathbf{2 0 1 1}$ \\
\hline Frecuencia de anemia en < de 3 años & 56,8 & 50,4 & 50,3 & 39,7 \\
CRED completo en < 3 años & 24,0 & 27,7 & 40,0 & 47,3 \\
Vacunas completas en < 3años & 64,3 & 54,8 & 61,8 & 72,7 \\
Suplemento de hierro en < 3 años & 12,3 & 14,1 & 18,4 & 17,0 \\
Suplemento de hierro en gestantes & 74,9 & 80,1 & 86,1 & 87,8 \\
\hline Proporción de hogares & 81,8 & 83,3 & 85,3 & 87,6 \\
con saneamiento básico & 24,0 & 17,2 & 18,3 & 17,8 \\
Frecuencia de IRA en < 3 años & 17,4 & 18,0 & 18,9 & 17,6 \\
\hline Frecuencia de EDA en < 3 años & 68,7 & 68,5 & 68,3 & 65,9 \\
\hline Lactancia materna exclusiva & 8,4 & 7,1 & 8,0 & 7,1 \\
\hline Bajo peso al nacer & & & & \\
\hline
\end{tabular}

Fuente: Instituto Nacional de Estadística e Informática.

la implementación de políticas públicas, la adopción de herramientas de gestión sencillas para los gobiernos locales, diseñar intervenciones flexibles y adecuadas al tipo de población y territorio específico, promover la articulación entre gobiernos regionales y locales, y facilitar el acceso a recursos para proyectos de inversión social (11).

\section{PROGRAMA ARTICULADO NUTRICIONAL}

Desde el año 2008, se viene implementando en el Perú, el Presupuesto por Resultados, conjunto de programas presupuestales que dirigen la asignación de recursos a productos con probada eficacia para conseguir resultados sobre los principales problemas que afectan al ciudadano. Uno de dichos programas presupuestales es el Programa Articulado Nutricional (PAN), el cual tiene como objetivo la disminución de la prevalencia de desnutrición crónica infantil en los niños menores de cinco años.

El análisis de impacto del Programa Articulado Nutricional demuestra que, bajo la aplicación de técnicas no experimentales de evaluación, este se asocia a una reducción de la probabilidad de ser desnutrido y un aumento de probabilidades de tener controles de crecimiento y vacunas completas en cohortes de nacimiento posteriores al año 2008 , año de inicio del programa ${ }^{(12)}$. Asimismo, se puede observar una mejora sostenida entre los años 2007-2011 de los indicadores de resultados intermedios y productos identificados por el PAN, con excepción del indicador de lactancia materna exclusiva (Tabla 1) ${ }^{(1,8)}$.

\section{LA NUEVA ETAPA}

Como lo demuestran las experiencias revisadas, se han logrado avances en la reducción de la desnutrición crónica infantil. El Plan Bicentenario estableció como meta alcanzable al 2021 una prevalencia de $5 \%$ de desnutrición crónica infantil (13). El Gobierno ha establecido al 2016, alcanzar como meta reducir la desnutrición crónica infantil en 13 puntos porcentuales para el año $2016{ }^{(14-16)}$. Es decir, teniendo como base la prevalencia de desnutrición crónica infantil para el año 2010 (23\%), se espera que para el 2016, esta sea de $10 \%$.

La Estrategia Sanitaria de Alimentación y Nutrición Saludable (ESANS) es presidida por el Instituto Nacional de Salud. Fue creada con la finalidad de contribuir a mejorar el estado alimentario nutricional de la población peruana, a través de acciones integrales de salud, alimentación y nutrición, priorizando los grupos vulnerables según etapa de vida, niveles de pobreza y estado de exclusión, a través del fortalecimiento de capacidades, generación de evidencias en alimentación y nutrición, asistencia técnica, fortalecimiento de los sistemas de información, supervisión y evaluación de acciones y de la implementación de la normatividad en alimentación y nutrición ${ }^{(17,18)}$.

En el ámbito de la investigación se realizaron esfuerzos importantes en el establecimiento de prioridades nacionales y regionales de investigación en salud para

Tabla 2. Agenda Nacional de Investigación en Desnutrición Infantil 2011-2014.

\section{Líneas de investigación priorizadas}

1. Impacto y costo efectividad de intervenciones orientadas a reducir la desnutrición infantil.

2. Inicio de la alimentación complementaria: cantidad, tipo, consistencia y calidad de los alimentos consumidos.

3. Prácticas de alimentación durante la enfermedad del niño.

4. Barreras y facilitadores socioculturales de la mejora de prácticas en alimentación Infantil, con énfasis en poblaciones andinas y amazónicas.

5. Evaluación de intervenciones en alimentación y cuidado de la gestante.

6. Determinantes de los comportamientos alimentarios nutricionales en la mujer gestante.

7. Evaluación integral de las intervenciones con micronutrientes.

8. Identificación de las causas y consecuencias de la deficiencia de hierro y otros micronutrientes en grupos vulnerables.

9. Identificación de los aspectos sociales y culturales que condicionan la alimentación y nutrición en la familia.

10. Evaluación del impacto de las políticas, programas y proyectos productivos sobre la seguridad alimentaria y nutricional.

11. Identificación de los factores que mejoran la articulación de la gestión pública en los programas y proyectos sociales en favor de la seguridad alimentaria y nutricional

12. Evaluación del costo efectividad de las intervenciones en nutrición desarrollados en los servicios de salud.

Fuente: Instituto Nacional de Salud 2011 
el periodo 2010-2014, bajo un enfoque articulador y participativo ${ }^{(19)}$. Como parte de este esfuerzo, se ha elaborado y aprobado la Agenda Nacional de Investigación en Desnutrición Infantil, con la participación de diferentes instituciones expertas en el tema, en la cual se establecen líneas principales de trabajo, que se muestran en la Tabla $2{ }^{(20)}$.

\section{CONCLUSIÓN}

En el país se han desarrollado importantes intervenciones que han contribuido a lograr una fuerte reducción de la desnutrición crónica infantil. Sin embargo, para continuar con el descenso de la desnutrición crónica se requiere realizar intervenciones en salud y nutrición con evidencias científicas, para el diseño de políticas públicas, el fortalecimiento de las capacidades de recursos humanos en salud y la articulación de los diferentes niveles de gobierno.

Fuentes de financiamiento: este trabajo fue realizado como una de las actividades laborales del autor en el Instituto Nacional de Salud, Lima, Perú.

Conflictos de interés: el autor declara ser parte del Centro Nacional de Alimentación y Nutrición del Instituto Nacional de Salud, responsable de la ejecución de algunas actividades descritas en el presente artículo.

\section{REFERENCIAS BIBLIOGRÁFICAS}

1. Instituto Nacional de Estadística e Informática (INEI). Encuesta Demográfica y de Salud Familiar ENDES 2011 Informe principal. Lima: INEI; 2011.

2. World Health Organization. Nutrition Landscape Information System (NLIS). Country profile indicators: interpretation guide. Geneva: WHO; 2010.

3. Fondo de las Naciones Unidas para la Infancia. Estado Mundial de la Infancia 1998. Ginebra: UNICEF; 1998.

4. Smith L, Haddad L. Overcoming child malnutrition in developing countries: past achievements and future choices. Washington: International Food Policy Research Institute; 2000

5. López N, Alí V, Rojas C. Acciones efectivas para reducir la desnutrición crónica. Evidencias del cambio en zonas rurales del Perú 2003-2004. Lima: CARE Perú; 2005.

6. Victora C, Adair L, Fall C, Hallal P, Martorell R, Richter L, et al. Maternal and child undernutrition: consequences for adult health and human capital. Lancet. 2008;371(9609):340-57.

7. Instituto Nacional de Estadística e Informática. Encuesta Demográfica y de Salud Familiar ENDES 1996 Informe principal. Lima: INEI; 1996.

8. Instituto Nacional de Estadística e Informática. Indicadores de Resultados de los Programas estratégicos 2010. Lima: INEI; 2011.

9. Perú, Presidencia del Consejo de Ministros (PCM). Aprueban la Estrategia Nacional denominada CRECER que establece la intervención articulada de entidades de Gobierno Nacional, Regional y Local vinculadas con la lucha contra la desnutrición crónica infantil. DS 055-2007-PCM. Lima: PCM; 2007. (1 de julio de 2007)

10. Mejía A. Análisis del éxito en la lucha contra la Desnutrición crónica en el Perú: estudio de caso preparado para CARE. Lima: CARE Perú; 2011.

11. Perú, Presidencia del Consejo de Ministros. Estrategia Nacional CRECER. Memoria 2007-2011. Lima: PCM; 2011.

12. Cruzado V. Análisis del impacto del Programa Presupuestal Articulado Nutricional sobre la desnutrición crónica en niños menores de 5 años. Lima: Ministerio de Economía y Finanzas; 2012.

13. Centro Nacional de Planeamiento Estratégico (CEPLAN). Plan Bicentenario: El Perú hacia el Siglo XXI. Lima: CEPLAN; 2011.

14. Gana Perú. Lineamientos centrales de política económica y social para un gobierno de concentración nacional. Campaña Ollanta Presidente: segunda vuelta [hoja de ruta]. Lima: Gana Perú: 2011.

15. Perú, Inversión en la Infancia. Desnutrición crónica infantil cero en el 2016: una meta alcanzable [Resumen Ejecutivo]. Lima; 2012.

16. Perú, Presidencia del Consejo de Ministros. Exposición de la Política General del Gobierno y Principales Medidas de Gestión 2012-2016. Lima: PCM; 2012.
17. Perú, Ministerio de Salud (MINSA). Establecen las Estrategias Sanitarias Nacionales del ministerio y sus respectivos órganos responsables. RM 771-2004/ MINSA. Lima: MINSA; 2004.

18. Perú, Ministerio de Salud. Reestructuran la organización y dependencia funcional de las Estrategias Sanitarias $\mathrm{Na}$ cionales del Ministerio de Salud. RM 525-2012/MINSA. Lima: MINSA; 2012.

19. Caballero P, Yagui M, Espinoza M, Castilla T, Granados A, Velásquez A, et al. Prioridades regionales y nacionales de investigación en Salud, Perú 20102014: Un proceso con enfoque participativo y descentralista. Rev Peru Med Exp Salud Pública. 2010;27(3):398411.

20. Instituto Nacional de Salud (INS). Aprobar la agenda Nacional de Investigación en Desnutrición Infantil para el periodo 2011-2014. RJ 175-2011-JOPE/INS. Lima: INS; 2011.

Correspondencia: José Ramón Sánchez Abanto.

Dirección: Jr. Tizón y Bueno 276, Lima 11, Perú.

Teléfono: (511) 999373783.

Correo electrónico:jsanchez@ins.gob.pe 